\title{
Around stable forking
}

\author{
by \\ Byunghan Kim (Cambridge, MA) and A. Pillay (Urbana, IL) \\ Dedicated to the memory of Jerzy Eoś
}

\begin{abstract}
We discuss various conjectures and problems around the issue of when and whether stable formulas are responsible for forking in simple theories. We prove that if the simple theory $T$ has strong stable forking then any complete type is a nonforking extension of a complete type which is axiomatized by instances of stable formulas. We also give another treatment of the first author's result which identifies canonical bases in supersimple theories.
\end{abstract}

1. Introduction and preliminaries. In the past few years various conjectures have been made concerning the relationship between simple theories and stable theories. The general thrust is that in a simple theory $T$ forking should be accounted for by some kind of "stable fragment" of $T$. These issues were raised in discussions between Hart, Kim and Pillay in the Fields Institute in the autumn of 1996, but it is quite likely that others have also formulated such problems. The purpose of this paper is to clarify some of these questions and conjectures as well as to prove some relations between them. The theory of local stability, namely the study of $\phi(x, y)$-types where $\phi(x, y)$ is a stable formula, will play an important role.

The present paper is closely related to the first author's paper [5], where some positive results are obtained for supersimple theories and simple 1based theories. One of the properties we will consider is "stable forking"; if a type $p(x) \in S(M)$ forks over a subset $A$ of $M$ then this should be witnessed by an instance of a stable formula. All known simple theories have this property, and the "stable forking conjecture" says that all simple theories have this property. We introduce another property, strong stable forking: if $p(x) \in S(B)$ forks over a set $A$, where $A$ is not necessarily con-

2000 Mathematics Subject Classification: Primary 03C45.

Research supported by NSF grants. 
tained in $B$, then this is witnessed by an instance of a stable formula in $p$. We show that if $T$ has strong stable forking, then up to nonforking, any complete type is axiomatized by instances of stable formulas (generalizing a result from [5]). We point out that pseudofinite fields do not have strong stable forking. In Section 3 we introduce the notions of canonical formulas and $p$-stable formulas, and with this notation we give a fast account of one of the main results from [5].

Thanks to Zoe Chatzidakis for some helpful comments, and to Ludomir Newelski for pointing out some errors in an earlier draft.

Throughout, $T$ will denote a complete theory in a language $L$, and $\bar{M}$ will be a big saturated model of $T$ in which we shall work. In fact, we will work in $\bar{M}^{\mathrm{eq}}$ and $L^{\mathrm{eq}}$. By $x, y, \ldots$ we will denote (finite) tuples of variables, or equivalently single variables from $L^{\mathrm{eq}}$, unless we say otherwise. Similarly $A, B, \ldots$ will denote (small) subsets of $\bar{M}^{\text {eq }}$ unless we say otherwise. In fact sometimes we will want to talk of hyperimaginary parameters and we will make this explicit. Forking is defined as usual: a formula $\phi(x, b)$ divides over $A$ if there is an $A$-indiscernible sequence $\left(b_{i}: i \in \omega\right)$ of realizations of $\operatorname{tp}(b / A)$ such that $\left\{\phi\left(x, b_{i}\right): i<\omega\right\}$ is inconsistent, and a partial type $\Sigma(x)$ forks over $A$ if $\Sigma(x)$ implies a finite disjunction of formulas each of which divides over $A$.

We will be talking at various times about complete types $p(x)$ being axiomatized by certain partial types $\Sigma(x)$. By this we mean that $\Sigma(x)$ and $p(x)$ have the same set of realizations. If $p(x) \in S(A)$, then $\Sigma(x)$ may or may not consist of formulas over $A$ (with parameters from $A$ ). In [2] we made sense out of $\operatorname{tp}(a / e)$ where $e$ is a hyperimaginary, as a nonuniquely defined partial type whose set of realizations is precisely the orbit of $a$ under $\operatorname{Aut}_{e}(\bar{M})$. Again we will say that $\Sigma(x)$ axiomatizes $\operatorname{tp}(a / e)$ if both partial types have the same set of realizations. The situation when $\Sigma(x)$ can be chosen to consist of formulas which are over $e$ (namely, invariant under Aut $_{e}(\bar{M})$ or with canonical parameter in $\bar{M}^{\mathrm{eq}}$ in $\left.\mathrm{dcl}(e)\right)$ is interesting: for example, if for every hyperimaginary $e$ and tuple $a$ from $\bar{M}^{\text {eq }}, \operatorname{tp}(a / e)$ can be axiomatized by a set of formulas over $e$, then $T$ has elimination of hyperimaginaries.

We assume acquaintance with the basic results and machinery concerning stable theories $([8])$ and simple theories ([4], [6] and [2]). In particular, we assume knowledge of the ranks $R(-, \phi, \omega)$ (from [9]). However, so as to fix notation we recall some things. In particular, the treatment of "local stability" theory comes from Chapter 1 of [8]: we are situated in a saturated structure $\bar{M}, \phi(x, y)$ is a stable formula, and we are concerned with complete $\phi$-types and their nonforking (or forking) extensions, where forking is meant in the sense of the ambient structure $\bar{M}$. 
Definition 1.1. We call an $L$-formula $\phi(x, y)$ stable if $\phi(x, y)$ does not have the order property in $\bar{M}$, namely there do not exist $a_{i}, b_{i}$ for $i<\omega$ such that $=\phi\left(a_{i}, b_{j}\right)$ iff $i<j$.

Definition 1.2. Let $\phi(x, y)$ be an $L$-formula. By an instance of $\phi$ we mean a formula $\phi(x, b)$ for some $b \in \bar{M}$. By a complete $\phi(x, y)$-type over a set $A$ we mean a maximal consistent set of formulas $\psi(x)$ which have parameters from $A$ and are equivalent to finite Boolean combinations of instances of $\phi(x, y)$. We let $\operatorname{tp}_{\phi}(a / A)$ denote the (complete) $\phi$-type of $a$ over $A$.

REMARK 1.3. Suppose that $\psi(x, b)$ is equivalent to a Boolean combination of instances of stable formulas $\phi_{i}\left(x, y_{i}\right)$. Then for some $\chi(z) \in \operatorname{tp}(b / \emptyset)$, the formula $\psi(x, z) \wedge \chi(z)$ is stable.

FACT 1.4. Let $\phi(x, y)$ be stable and let $p(x)$ be a complete $\phi$-type over a model M. Then:

(i) $p$ is definable (i.e. there is a formula $\psi(y)$ over $M$ such that for all $b \in M, \mid=\psi(b)$ iff $\phi(x, b) \in p(x))$. We call $\psi(y)$ the $\phi$-definition of $p$.

(ii) Let $c$ be a canonical parameter for the $\phi$-definition of $p$. Then $p(x)$ does not fork over $c$. Moreover, for any model $N$ containing $c, p \mid c$ has a unique nonforking extension to a complete $\phi$-type $q(x)$ over $N$, and the $\phi$ definition of such a $q$ is the same as the $\phi$-definition of $p$.

(iii) Let $c$ be as in (ii), and $A \subset M$. Then $p$ does not fork over $A$ iff $c \in \operatorname{acl}(A)$.

REMARK 1.5. Let $p(x), \phi(x, y)$ and $c$ be as in Fact 1.4. We call $c$ the canonical base of $p$.

FACT 1.6. Let $\phi(x, y)$ be stable. Let $p(x) \subseteq q(x)$ be complete $\phi$-types over $A \subseteq B$ respectively. Then $q$ does not fork over $A$ iff $R(q, \phi(x, y), \omega)=$ $R(p, \phi(x, y), \omega)$. Moreover, $\operatorname{mult}(p, \phi, \omega)=1$ iff $p$ is stationary (for each $C \supseteq A, p$ has a unique nonforking extension to a complete $\phi(x, y)$-type over $C)$.

We now review the theory of canonical bases in simple theories. Let us now assume $T$ to be simple. Let $e$ be a hyperimaginary and $p(x) \in S(e)$. We call $p(x)$ an amalgamation base if whenever $p_{1}, p_{2}$ are nonforking extensions of $p$ over hyperimaginaries $d, f$ where $e \in \operatorname{dcl}(d) \cap \operatorname{dcl}(f)$ and $d$ is independent of $f$ over $e$, then $p_{1}(x) \cup p_{2}(x)$ does not fork over $e$. Any complete type over a model is an amalgamation base, as well as a complete type over a bounded-closed set (of hyperimaginaries). For an amalgamation base $p(x) \in S(e)$, the class $\mathbf{P}_{p}$ of complete types over $\bar{M}$ is defined inductively by: any nonforking extension of $p(x)$ to a complete type $q(x) \in S(\bar{M})$ is in $\mathbf{P}_{p}$. Suppose $q \in \mathbf{P}_{p}, q$ does not fork over $b$ and $q \mid b$ is an amalgamation 
base. Then any nonforking extension of $q \mid b$ (to a complete type over $\bar{M}$ ) is in $\mathbf{P}_{p}$.

FACT 1.7. Let $p(x) \in S(e)$ be an amalgamation base.

(i) There is a hyperimaginary $e^{\prime}$ such that any automorphism $f$ of $\bar{M}$ fixes $e^{\prime}$ iff it fixes $\mathbf{P}_{p}$ setwise. This $e^{\prime}$ is called the canonical base of $p, \mathrm{Cb}(p)$, and is unique up to interdefinability.

(ii) Let $e^{\prime}=\mathrm{Cb}(p)$. Then $e^{\prime} \in \operatorname{dcl}(e)$. For any $f \in \operatorname{dcl}(e)$, $p$ does not fork over $f$ if and only if $e^{\prime} \in \operatorname{bdd}(f)$.

We point out the coherence of the two notions of canonical base (simple theories and local stability).

REMARK 1.8. Suppose $p(x) \in S(e)$ is an amalgamation base. Let $\phi(x, y)$ be a stable formula. Then all $q(x) \in \mathbf{P}_{p}$ have the same $\phi(x, y)$-type. (Namely, if $q_{i} \in \mathbf{P}_{p}$ for $i=1,2$ then $q_{1}\left|\phi(x, y)=q_{2}\right| \phi(x, y)$.) If $c$ is the canonical base for this complete $\phi(x, y)$-type over $\bar{M}$, then $c \in \operatorname{dcl}(\mathrm{Cb}(p))$.

2. Stable forking and strong stable forking. In this section, $T$ will be a simple theory. Again, $A, B, \ldots$ will be (small) subsets of $\bar{M}^{\mathrm{eq}}$ unless otherwise stated.

Definition 2.1. (i) We say that $T$ has stable forking if whenever $q(x) \in$ $S(M)$ is a complete type over a model $M, A \subseteq M$ and $q(x)$ forks over $A$ then there is some $\psi(x, b) \in q(x)$ such that $\psi(x, y) \in L$ is stable, and $\psi(x, b)$ forks over $A$.

(ii) We say that $T$ has strong stable forking if whenever $q(x) \in S(B)$ and $A$ is arbitrary (not necessarily contained in $B$ ) and $q(x)$ forks over $A$ then there is some $\psi(x, b) \in q(x)$ such that $\psi(x, y)$ is stable and $\psi(x, b)$ forks over $A$.

REMARK 2.2. (i) Every known example of a simple theory has stable forking. We will see below that any 1-based simple theory has strong stable forking, but that pseudofinite fields, for example, do not have strong stable forking.

(ii) $T$ has strong stable forking iff whenever $\Sigma(x)$ is a partial type over a set $B$ which forks over a set $A$ then there is a stable formula $\psi(x, y) \in L$ and $b \in B$ such that $\psi(x, b)$ is implied by $\Sigma(x)$ and $\psi(x, b)$ forks over $A$.

Proof of (ii). Assume $T$ has strong stable forking. Let $\Sigma(x)$ be our partial type over $B$ which forks over $A$. Then every completion of $\Sigma(x)$ over $B$ forks over $A$. By our assumptions and compactness there is a finite disjunction of formulas $\psi_{i}\left(x, b_{i}\right)$, implied by $\Sigma(x)$, where each $b_{i}$ is in $B$, $\psi_{i}\left(x, y_{i}\right)$ is stable, and each $\psi_{i}\left(x, b_{i}\right)$ forks over $A$. By Remark 1.3 , we get the desired conclusion. 
Our first (easy) observation is:

Proposition 2.3. The following are equivalent:

(i) $T$ has stable forking.

(ii) Whenever $p(x)$ is an amalgamation base and $C$ is the set of $\phi$ definitions of $\mathbf{P}_{p}$ as $\phi(x, y)$ ranges over stable formulas, then $p(x)$ does not fork over $C$.

Proof. (i) $\Rightarrow$ (ii). Assume (i). Let $p(x)$ be an amalgamation base. Let $p^{\prime}(x)$ be some nonforking extension of $p(x)$ to a model $M$. Note that $\mathbf{P}_{p}=$ $\mathbf{P}_{p^{\prime}}$. Let $C$ be as in (ii). It is enough to show that $p^{\prime}(x)$ does not fork over $C$. Suppose otherwise. So by stable forking there is some stable $\phi(x, y) \in L$ and $b \in M$ such that $\phi(x, b) \in p^{\prime}(x)$ and $\phi(x, b)$ forks over $C$. In particular, $p^{\prime}(x) \mid \phi(x, y)$ forks over $C$. By 1.4 the $\phi(x, y)$-definition of $p^{\prime}(x)$ is not over $C$, contradicting the definition of $C$.

(ii) $\Rightarrow$ (i). Assume (ii). Let $p(x) \in S(M)$ and suppose $p(x)$ forks over $A \subseteq M$. Let $C$ be as in (ii) for $p(x)$. By 1.8, $C \subseteq \operatorname{dcl}(\mathrm{Cb}(p))$, and by our assumption and $1.7, \mathrm{Cb}(p) \subseteq \operatorname{bdd}(C)$. $\mathrm{By} 1.7, \mathrm{Cb}(p)$ is not included in $\operatorname{bdd}(A)$, whereby $C$ is not contained in $\operatorname{acl}(A)$. Thus for some stable formula $\phi(x, y)$, the $\phi$-definition of $p(x)$ is not over $\operatorname{acl}(A)$. By $1.4, p \mid \phi$ forks over $A$, so some formula $\psi(x) \in p \mid \phi$ forks over $A$. By $1.3, \psi(x)$ is an instance of a stable formula. We have proved (i).

REMARK 2.4. $T$ has stable forking iff for any $A \subseteq B$ and $q(x) \in S(B)$, if $q$ forks over $A$ then $q(x)$ contains an instance $\psi(x, b)$ of a stable formula such that $\psi(x, b)$ forks over $A$.

Proof. Suppose $q(x)$ forks over $A$. Let $M$ be a model containing $B$ and let $q^{\prime}(x) \in S(M)$ be a nonforking extension of $q(x)$. So $q^{\prime}(x)$ forks over $A$. Assuming stable forking, there is a stable formula $\phi(x, y)$ such that $q^{\prime} \mid \phi$ forks over $A$. But then the restriction of $q^{\prime} \mid \phi$ to $B$ forks over $A$, and if this is witnessed by $\psi(x)$, then $\psi(x)$ is as required.

Proposition 2.5. Suppose T has strong stable forking.

(i) Let $p(x)$ be an amalgamation base over $B$ (where $B$ may consist of hyperimaginaries). Let $C$ be the set of $\phi$-definitions of $\mathbf{P}_{p}$ as $\phi(x, y)$ ranges over stable formulas. Then ( $p$ does not fork over $C$ and) $p \mid C$ is axiomatized by instances of stable formulas with parameters in $C$.

(ii) Let $p(x)$ be a complete type over $B$ (where $B$ may consist of hyperimaginaries). Then there is $A \subset \bar{M}^{\mathrm{eq}}$ such that $A \subseteq \operatorname{dcl}(B)$ and $p \mid A$ is axiomatized by instances of stable formulas with parameters from $A$.

Proof. (i) For each stable formula $\phi(x, y)$, let $\psi_{\phi}\left(y, e_{\phi}\right)$ be a $\phi(x, y)$ definition of $\mathbf{P}_{p}$. We may assume that $e_{\phi}$ is a canonical parameter for $\psi_{\phi}\left(y, e_{\phi}\right)$. Let $e$ be the infinite tuple consisting of all the $e_{\phi}$ (so $e$ is an 
enumeration of $C$ ). By Proposition 2.3 (as clearly $T$ has stable forking), $p(x)$ does not fork over $e$. Write $p \mid e$ as $p^{0}(x, e)$ for some complete type $p^{0}(x, z)$. We will show that $p^{0}(x, e)$ is axiomatized by instances of stable formulas over $e$. Let $p^{1}(x, z)$ be the set of stable $L$-formulas in $p^{0}(x, z)$ (namely, $p^{1}(x, z)$ consists of the set of formulas $\psi\left(x, z^{\prime}\right) \in p^{0}(x, z)$ such that $z^{\prime}$ is a finite subtuple of $z$ and $\psi\left(x, z^{\prime}\right)$ is stable).

Claim. $p^{1}(x, e)$ axiomatizes $p^{0}(x, e)$.

Proof. Let $a$ realize $p^{1}(x, e)$. We want to prove that $a$ realizes $p^{0}(x, e)$. Note that $\operatorname{tp}(a)=p(x) \mid \emptyset$ (as every formula $\psi(x) \in p(x) \mid \emptyset$ is stable and hence in $\left.p^{1}(x, z)\right)$. Thus $p^{0}(a, z)$ is consistent.

Subclaim (a). $p^{0}(a, z)$ does not fork over e.

Proof. Suppose otherwise. Then some restriction $q\left(a, z_{0}\right)$ of $p^{0}(a, z)$ to a finite subtuple $z_{0}$ of variables forks over $e$. By strong stable forking, applied to $q\left(a, z_{0}\right)$ and $e$, there is a formula $\chi\left(a, z_{0}\right) \in q\left(a, z_{0}\right)$ which forks over $e$ and such that $\chi\left(x, z_{0}\right)$ is stable. Let $e_{0}$ be the finite subtuple of $e$ corresponding to $z_{0}$. So $\chi\left(x, e_{0}\right) \in p^{0}(x, e)$. But $\chi\left(x, e_{0}\right)$ is an instance of the stable formula $\chi\left(x, z_{0}\right)$, hence $\chi\left(x, e_{0}\right) \in p^{1}(x, e)$ and so $=\chi\left(a, e_{0}\right)$. Namely, $\chi\left(a, z_{0}\right)$ is satisfiable by a tuple from $e$, contradicting the fact that $\chi\left(a, z_{0}\right)$ forks over $e$. We have proved Subclaim (a).

By Subclaim (a) we can choose $e^{\prime}$ realizing $p^{0}(a, z)$ such that $\operatorname{tp}\left(e^{\prime} / a, e\right)$ does not fork over $e$. By symmetry, $\operatorname{tp}\left(a / e, e^{\prime}\right)$ does not fork over $e$.

Note that $\operatorname{tp}\left(e^{\prime}\right)=\operatorname{tp}(e)$ so we can write $e^{\prime}=\left(e_{\phi}^{\prime}: \phi(x, y)\right.$ stable $)$

Subclaim (b). $e_{\phi}^{\prime}=e_{\phi}$ for all stable $\phi(x, y)$.

Proof. Fix a stable formula $\phi(x, y)$. Note that $\operatorname{tp}_{\phi}\left(a / e_{\phi}^{\prime}\right)$ is the conjugate of $\operatorname{tp}_{\phi}\left(a / e_{\phi}\right)$ (under an automorphism taking $e_{\phi}$ to $e_{\phi}^{\prime}$ ), so they have the same $R(-, \phi, \omega)$-rank, $n$ say. By $(*)$ and the choice of $e_{\phi}, \operatorname{tp}_{\phi}\left(a / e, e^{\prime}\right)$ does not fork over $e_{\phi}$, so by 1.6 , has $R(-, \phi, \omega)$-rank $n$ as well. By 1.6 again, $\operatorname{tp}_{\phi}\left(a / e, e^{\prime}\right)$ does not fork over $e_{\phi}^{\prime}$. We have shown that $\operatorname{tp}_{\phi}\left(a / e_{\phi}\right)$ and $\operatorname{tp}_{\phi}\left(a / e_{\phi}^{\prime}\right)$ have a common nonforking extension (to a complete $\phi$-type over a model). By Fact $1.4, \psi_{\phi}\left(y, e_{\phi}\right)$ must be equivalent to $\psi_{\phi}\left(y, e_{\phi}^{\prime}\right)$, and thus $e_{\phi}=e_{\phi}^{\prime}$, as required.

From Subclaim (b) we see that $(a, e)$ realizes $p^{0}(x, z)$, namely $a$ realizes $p^{0}(x, e)$. This completes the proof of the Claim and so part (i) of the proposition.

(ii) Let $p(x) \in S(B)$. Let $B^{\prime}=\operatorname{bdd}(B)$, and $p^{\prime}$ an extension of $p$ to a complete type over $B^{\prime}$. Let $C$ be as in part (i). Note that $C \subseteq \operatorname{acl}(B)$. Let $C_{0} \subseteq \bar{M}^{\text {eq }}$ be the set of codes for (finite) sets of $B$-conjugates of finite tuples from $C$. Then $C_{0} \subseteq \operatorname{dcl}(B) \cap \operatorname{acl}(A)$. For each instance $\psi(x)$ of a stable 
formula in $p^{\prime} \mid C$, let $\psi^{\prime}(x)$ be the disjunction of the finite set of $B$-conjugates of $\psi$. Then $\psi^{\prime}(x)$ is an instance of a stable formula and $\psi^{\prime}(x)$ is over $C_{0}$. Let $p^{\prime \prime}$ be the set of all such $\psi^{\prime}(x)$. We claim that $p^{\prime \prime}$ axiomatizes $p^{\prime} \mid C_{0}\left(=p \mid C_{0}\right)$. Let $d$ realize $p^{\prime \prime}$. For each $\psi(x, c) \in p^{\prime} \mid C$ there is an elementary permutation $\sigma$ of $\operatorname{acl}(B)$ over $B$ such that $\psi(d, \sigma(c))$ holds. A König's Lemma argument shows that we may assume all these $\sigma$ to be the same, and thus $\sigma^{-1}(d)$ realizes $p^{\prime} \mid C$. But $C_{0}$ is contained in $\operatorname{dcl}(B)$ and $\operatorname{sotp}\left(d / C_{0}\right)=p^{\prime}\left|C_{0}=p\right| C_{0}$. Note that $p^{\prime}$ and thus $p$ does not fork over $C_{0}$. This proves (ii).

REMARK 2.6. (i) A theory is stable if and only if every complete type over any set $A$ of parameters is axiomatized by instances of stable formulas over $A$.

(ii) In the light of (i), we can think of the conclusion of Proposition 2.5 as saying that $T$ is stable up to nonforking.

Proof of (i). Suppose the right hand side condition holds. Let $\phi(x, y)$ be an arbitrary $L$-formula. If $\phi(x, y)$ is unstable, we can find an indiscernible sequence $\left(\left(a_{i}, b_{i}\right): i<\omega\right)$ such that $\models \phi\left(a_{i}, b_{j}\right)$ iff $i<j$. Let $p(x, y)=$ $\operatorname{tp}\left(a_{0}, b_{1}\right)$. Now $p\left(x, b_{1}\right)$ is by assumption axiomatized by stable formulas over $b_{1}$. Thus there is some stable $\psi(x, y) \in L$ such that

(a) $\models \psi\left(x, b_{1}\right) \rightarrow \phi\left(x, b_{1}\right)$, and

(b) $\models \psi\left(a_{0}, b_{1}\right)$.

By indiscernibility and (b), we have $=\psi\left(a_{i}, b_{j}\right)$ whenever $i<j$.

On the other hand by (a) and the assumptions on $\left(\left(a_{i}, b_{i}\right): i<\omega\right)$ we have $=\neg \psi\left(a_{i}, b_{j}\right)$ whenever $i \geq j$.

Thus $=\psi\left(a_{i}, b_{j}\right)$ iff $i<j$, contradicting stability of $\psi(x, y)$.

REMARK 2.7. (i) In [5] it was shown that 1-based theories which admit elimination of hyperimaginaries satisfy the conclusion of Proposition 2.5.

(ii) Does the converse to Proposition 2.5 hold?

Proposition 2.8. Suppose $T$ is 1-based in the following sense: for any $A, B \subseteq \bar{M}^{\mathrm{eq}}, A$ is independent of $B$ over $\operatorname{acl}^{\mathrm{eq}}(A) \cap \operatorname{acl}^{\mathrm{eq}}(B)$. Then $T$ has strong stable forking.

Proof. Suppose $p(x) \in S(B)$ forks over $A$ (where $A$ need not be a subset of $B$ ). We want to show that there is an instance of a stable formula in $p$ which forks over $A$. We may clearly assume $B$ to be algebraically closed. Let $c$ realise $p(x)$. Let $C=\operatorname{acl}^{\mathrm{eq}}(c) \cap B$. So $p(x)$ does not fork over $C$. On the other hand, $p(x) \mid C=\operatorname{tp}(c / C)$ is clearly axiomatized by instances (over $C$ ) of stable formulas $\phi(x, y)$ which imply $y \in \operatorname{acl}(x)$. So it is enough to prove:

Claim. $p(x) \mid C$ forks over $A$.

Proof. Otherwise there is $c^{\prime}$ realizing $p(x) \mid C$ such that $c^{\prime}$ is independent of $C$ over $A$. As $C \subseteq \operatorname{acl}\left(c^{\prime}\right)$, it follows that $C \subseteq \operatorname{acl}(A)$. But then, any 
nonforking extension of $p(x)$ over $B \cup A$ does not fork over $C$ so does not fork over $A$, contradicting our hypothesis.

Note that by 2.3 , if $T$ has stable forking, $p(x)$ is an amalgamation base, and $C$ is the set of $\phi$-definitions for $\mathbf{P}_{p}$ as $\phi(x, y)$ ranges over stable formulas, then $C \subseteq \operatorname{dcl}(\mathrm{Cb}(p))$ and $\mathrm{Cb}(p) \subseteq \operatorname{bdd}(C)$. The question is left open regarding whether $\operatorname{dcl}(C)=\operatorname{dcl}(\operatorname{Cb}(p))$, even if we assume that Lascar strong types and strong types coincide over sets in $\bar{M}^{\mathrm{eq}}$. In a similar vein, if $T$ in addition has strong stable forking, is $p \mid \mathrm{Cb}(p)$ axiomatized by instances of stable formulas? As pointed out in [5] this is the case for $T$ 1-based.

We finish this section with an example which does not have strong stable forking.

REMARK 2.9. Let $T$ be (any completion of) the theory of pseudofinite fields. Then $T$ does not satisfy the conclusion of 2.5. In particular, $T$ does not have strong stable forking.

Sketch proof. Work in a saturated model $K$ of $T$. We will assume $\operatorname{char}(K)$ $\neq 2$. Let $a$ be (a canonical parameter for) a "generic" plane in $K^{3}$ (meaning precisely that $a$ is a generic 3 -tuple $\left(a^{1}, a^{2}, a^{3}\right)$ and the plane we are considering is defined by $z=a^{1} x+a^{2} y+a^{3}$ say). Let $b$ be a canonical parameter for a generic line on $a$, and let $c=\left(c^{1}, c^{2}, c^{3}\right.$ ) be a generic (over $a, b)$ point on $b$. Let $p(x, a)=\operatorname{tp}(c / a)$ and $q(x, b)=\operatorname{tp}(c / b)$. This choice can be made so that $c^{1}-a^{1}$ is a square. Using properties of pseudofinite fields, we can construct an indiscernible sequence $\left(c_{i}, a_{i}\right)$ in $\operatorname{tp}(c, a)$ such that the planes $c_{i}$ intersect in $b$ and are independent and indiscernible over $b$, each $c_{i}$ realizes $q(x, b), c_{i}$ realizes $p\left(x, a_{j}\right)$ if $i \leq j$ and for $i>j, c_{i}^{1}-a_{j}^{1}$ is not a square.

This shows that $p(x, a)$ cannot be axiomatized by instances of stable formulas. Note that if $A \subset \operatorname{dcl}(a)$ is such that $p(x, a)$ does not fork over $A$ then $A=\operatorname{dcl}(a)$, so the conclusion of 2.5 does not hold.

3. Canonical formulas and supersimple theories. The previous conventions remain in place. We introduce another property " $T$ has canonical formulas". We show it is a consequence of "stable forking + Lstp = stp". We also discuss the connection with " $p$-stable" formulas. In [5] the first author showed essentially that supersimple theories have canonical formulas and we finish the present paper with a fast proof of this.

Definition 3.1. (i) By a canonical type we mean a complete type $p(x)$ over $c$ such that $p(x)$ is an amalgamation base and $c=\mathrm{Cb}(p)$. (So possibly $c$ is a hyperimaginary.) 
(ii) Let $p(x)$ be an amalgamation base. We call $\phi(x, c)$ a canonical formula for $p(x)$ if $\phi(x, c)$ is in some $q(x) \in \mathbf{P}_{p}$ but whenever $\phi\left(x, c^{\prime}\right)$ is a conjugate of $\phi(x, c)$ which is not equivalent to $\phi(x, c)$ then $\phi\left(x, c^{\prime}\right)$ is in no $q(x) \in \mathbf{P}_{p}$.

(iii) We say that $T$ has canonical formulas if for every amalgamation base $p(x), \mathrm{Cb}(p)$ is interdefinable with the set of (canonical parameters of) canonical formulas for $p(x)$.

REMARK 3.2. (i) Let $p(x)$ be an amalgamation base, and $c=\mathrm{Cb}(p)$. Let $\phi(x, d)$ be any formula. Then $\phi(x, d)$ is a canonical formula for $p(x)$ iff [for all $d^{\prime}$ with $\operatorname{tp}\left(d^{\prime}\right)=\operatorname{tp}(d),(p(x) \mid c) \cup\left\{\phi\left(x, d^{\prime}\right)\right\}$ does not fork over $c$ iff $\phi\left(x, d^{\prime}\right)$ is equivalent to $\phi(x, d)]$. Moreover, if $\phi(x, d)$ is a canonical formula for $p(x)$ then $\phi(x, d)$ is over $c$ and in $p(x) \mid c$.

(ii) Suppose $T$ has canonical formulas. Then any canonical type $p(x)$ is axiomatized by the set of canonical formulas for $p(x)$. (The converse also holds.)

Proof. (i) is clear.

(ii) Let $p(x)$ be a canonical type, and let $d$ be the sequence of canonical parameters for canonical formulas in $p$. So (by hypothesis) we may assume that $p(x)$ is over $d$. Let $d^{\prime}=\left(d_{1}, \ldots, d_{n}\right)$ be a finite tuple from $d$. Let $\phi_{i}\left(x, d_{i}\right)$ be a canonical formula for $p(x)$ with canonical parameter $d_{i}$. Let $\psi\left(x, d^{\prime}\right)$ be any formula in $p(x) \mid d^{\prime}$ which implies each $\phi_{i}\left(x, d_{i}\right)(i=1, \ldots, n)$. Then clearly $\psi\left(x, d^{\prime}\right)$ is also a canonical formula for $p(x)$. (If $f$ is an automorphism of the universe which moves $d^{\prime}$ then $f$ moves some $d_{i}$, hence $\phi_{i}\left(x, f\left(d_{i}\right)\right) \cup p(x)$ forks over $d$, hence $\psi\left(x, f\left(d^{\prime}\right)\right) \cup p(x)$ forks over $d$.)

Lemma 3.3. Suppose $T$ has stable forking and Lstp $=$ stp over any set $A \subseteq \bar{M}^{\mathrm{eq}}$. Then $T$ has canonical formulas.

Proof. Let $p(x)$ be an amalgamation base, without loss of generality over a saturated model $M$. Fix a stable $L$-formula $\phi(x, y)$. Let $c_{\phi}=\mathrm{Cb}(p \mid \phi)=$ the $\phi(x, y)$-definition of $p(x)$. Let $p_{0}$ be the restriction of $p \mid \phi$ to $c_{\phi}$. Let $n=$ $R(p \mid \phi, \phi(x, y), \omega)$. By 1.6 there is a formula $\psi(x) \in p_{0}$ with $R(\psi(x), \phi(x, y), \omega)$ $=n$ and $\operatorname{mult}(\psi(x), \phi(x, y), \omega)=1$. It can be seen that an automorphism $f$ fixes $p(x) \mid \phi$ iff it fixes $\psi(x)$. Hence $\psi(x)$ is canonical for $p(x) \mid \phi$ so also for $p(x)$. Moreover, $c_{\phi}$ is interdefinable with the canonical parameter for $\psi(x)$. Let $C_{0}=\left\{c_{\phi}: \phi(x, y)\right.$ stable $\}$. By Proposition 2.3, $p(x)$ does not fork over $C_{0}$ and by 1.6, $C_{0} \subseteq \operatorname{dcl}(\mathrm{Cb}(p))$. As Lstp $=$ stp over $C_{0}, \mathrm{Cb}(p)$ is interdefinable with the set of all $E$-classes of $a$, where $a$ realizes $p$ and $E(x, y)$ is a $C_{0}$-definable finite equivalence relation. Let $E(x, y, c)$ be such, where $c$ is a finite tuple from $C_{0}$. Let $c=\left(c_{1}, \ldots, c_{r}\right)$, and by what we saw above let $\psi_{i}(x)$ be a formula over $c_{i}$ which is canonical for $p(x)$. Let $d$ be a canonical parameter for the $E$-class of $a$. Let $\chi(x, c, d)$ be the formula 
$\psi_{1}\left(x, c_{1}\right) \wedge \ldots \wedge \psi_{r}\left(x, c_{r}\right) \wedge$ " $d$ is the $E(x, y, c)$-class of $x$ ". It is then clear that $\chi(x, c, d)$ is canonical for $p(x)$ and that moreover $(c, d)$ is a canonical parameter for $\chi(x, c, d)$. $\operatorname{As~} \mathrm{Cb}(p)$ is the set of all such $(c, d)$, we have shown that $T$ has canonical formulas.

We recall another notion from [5], but we change notation slightly.

Definition 3.4. Let $p(x)$ be an amalgamation base. An $L$-formula $\phi(x, y)$ is said to be $p$-stable if all $q(x) \in \mathbf{P}_{p}$ have the same complete $\phi(x, y)$ type.

REMARK 3.5. If $\phi(x, y)$ is $p$-stable, then the common $\phi(x, y)$-type of all $q \in \mathbf{P}_{p}$ is definable. If $p$ is already over a model, this is just the $\phi$-definition of $p$.

Lemma 3.6. Suppose $T$ has canonical formulas. Then for any amalgamation base $p(x), \mathrm{Cb}(p)$ is the set of $\phi(x, y)$-definitions of $p$ as $\phi(x, y)$ ranges over p-stable formulas.

Proof. Let $\phi(x, c)$ be a canonical formula for $p$, where $c$ is assumed to be a canonical parameter for $\phi(x, c)$. Let $p_{0}$ be the restriction of $p$ to its canonical base. Let $r(y)=\operatorname{tp}(c)$. By assumption, if $\operatorname{tp}\left(c^{\prime}\right)=r(y)$ and $c^{\prime} \neq c$ then $p_{0}(x) \cup\left\{\phi\left(x, c^{\prime}\right)\right\}$ forks over $\mathrm{Cb}(p)$. By compactness, a finite amount $\psi(y)$ of $r(y)$ is responsible. Let $\psi^{\prime}(x, y)=\phi(x, y) \wedge \psi(y)$. Thus $\psi^{\prime}(x, c)$ is the unique instance of $\psi^{\prime}(x, y)$ which is in some type in $\mathbf{P}_{p}$. In particular $\psi^{\prime}(x, y)$ is $p$-stable and clearly $c$ is a canonical parameter for the $\psi^{\prime}(x, y)$ definition.

Questions. (1) Suppose that for every amalgamation base $p(x), \mathrm{Cb}(p)$ is the set of $\phi$-definitions of $p(\phi(x, y) p$-stable). $\mathrm{Is} p \mid \mathrm{Cb}(p)$ axiomatized by instances of $p$-stable formulas?

A stronger version is:

(2) With the same assumptions as (1), does $T$ have canonical formulas? Finally:

Proposition 3.7. Suppose $T$ is supersimple. Then $T$ has canonical formulas.

Proof. By [1], $T$ has elimination of hyperimaginaries. Let $p(x)$ be a canonical type. So $p(x)$ is a complete type over a (possibly infinite) tuple $c$ of imaginaries where $c=\mathrm{Cb}(p)$. By supersimplicity, let $c_{0}$ be a finite tuple from $c$ such that $p$ does not fork over $c_{0}$. By 1.7, $c \in \operatorname{acl}\left(c_{0}\right)$. Let $c_{1}$ be an arbitrary finite tuple from $c$ which includes $c_{0}$. We will show that $c_{1}$ is a canonical parameter for some canonical formula for $p$. As a matter of notation, if $c^{\prime}$ is a realization of $\operatorname{tp}(c)$, we let $c_{1}^{\prime}$ be the finite subtuple of $c^{\prime}$ corresponding to $c_{1}$. Also, we change notation by rewriting $p(x)$ as $p(x, c)$. 
Claim 1. Suppose $\operatorname{tp}\left(c^{\prime}\right)=\operatorname{tp}(c)$ and $c_{1}^{\prime} \neq c_{1}$. Then $p(x, c) \cup p\left(x, c^{\prime}\right)$ forks over $c$.

This is clear from properties of canonical types.

We may apply compactness to Claim 1 to find some $L$-formula $\phi(x, y)$, some $k<\omega$, some finite tuple $c_{2}$ from $c$ extending $c_{1}$ and a formula $\psi\left(x, c_{2}\right) \in$ $p(x, c)$ satisfying

CLAim 2. If $\operatorname{tp}\left(c_{2}^{\prime}\right)=\operatorname{tp}\left(c_{2}\right)$ and $c_{1}^{\prime} \neq c_{1}$ then

$$
D\left(\psi\left(x, c_{2}\right) \wedge \psi\left(x, c_{2}^{\prime}\right), \phi, k\right)<n
$$

where $n=D(p(x, c), \phi, k)$.

We may clearly assume that $D\left(\psi\left(x, c_{2}\right), \phi, k\right)=n$.

Note that $c_{2} \in \operatorname{acl}\left(c_{1}\right)$. Let $\chi(w, z)$ be an $L$-formula such that $\chi\left(w, c_{1}\right)$ isolates $\operatorname{tp}\left(c_{2} / c_{1}\right)$. Let $\psi^{\prime}(x, z)$ be the formula $\exists w(\psi(x, w) \wedge \chi(w, z))$. So $\psi^{\prime}\left(x, c_{1}\right)$ is equivalent to a finite disjunction of conjugates of $\psi\left(x, c_{2}\right)$. Thus $D\left(\psi^{\prime}\left(x, c_{1}\right), \phi, k\right)=n$ and Claim 2 implies

Claim 3. Whenever $c_{1}^{\prime} \neq c_{1}$ realizes $\operatorname{tp}\left(c_{1}\right)$ then

$$
D\left(\psi^{\prime}\left(x, c_{1}^{\prime}\right) \wedge \psi^{\prime}\left(x, c_{1}\right), \phi, k\right)<n .
$$

Now $\psi^{\prime}\left(x, c_{1}\right)$ is clearly in $p(x)$ and by Claim 3 , it is a canonical formula for $p(x)$ and also $c_{1}$ is a canonical parameter for $\psi^{\prime}\left(x, c_{1}\right)$. As $c_{1}$ was an essentially arbitrary finite tuple from $c$ we have proved the proposition.

REMARK 3.8. From 3.7 and 3.2(ii) we see that in a supersimple theory, any canonical type $p(x)$ is axiomatized by canonical formulas for $p(x)$ (equivalently, by instances of $p$-stable formulas). By Remark 2.9, these need not be stable formulas. For example, let $p(x, a)$ be as in the proof of 2.9. As shown there, the formula $\phi(x, y)$ : "the point $x=\left(x^{1}, x^{2}, x^{3}\right)$ is on the plane $y=\left(y^{1}, y^{2}, y^{3}\right)$, and $x^{1}-y^{1}$ is a square", is in $p(x, y)$ but is unstable. On the other hand, $\phi(x, a)$ is canonical for $p$.

\section{References}

[1] S. Buechler, A. Pillay and F. Wagner, Supersimple theories, J. Amer. Math. Soc. 14 (2000), 109-124.

[2] B. Hart, B. Kim and A. Pillay, Coordinatization and canonical bases in simple theories, J. Symbolic Logic 65 (2000), 293-309.

[3] E. Hrushovski, Simplicity and the Lascar group, preprint, 1997.

[4] B. Kim, Forking in simple unstable theories, J. London Math. Soc. 57 (1998), 257267.

[5] —, Simplicity, and stability in there, J. Symbolic Logic 66 (2001), 822-836.

[6] B. Kim and A. Pillay, Simple theories, Ann. Pure Appl. Logic 88 (1997), 149-164. 
[7] D. Lascar and A. Pillay, Hyperimaginaries and automorphism groups, J. Symbolic Logic 66 (2001), 127-143.

[8] A. Pillay, Geometric Stability Theory, Oxford Univ. Press, 1996.

[9] S. Shelah, Classification Theory, North-Holland, 2nd ed., 1990.

Department of Mathematics

MIT

Cambridge, MA 02138, U.S.A.

E-mail: bkim@math.mit.edu
Department of Mathematics University of Illinois at Urbana-Champaign Altgeld Hall, 1409 W. Green St. Urbana, IL 61801, U.S.A. E-mail: pillay@math.uiuc.edu

Received 1 December 1999;

in revised form 14 December 2000 